\title{
Acute effect of lactic acid on tumor-endothelial cell metabolic coupling in the tumor microenvironment
}

\author{
GUANQUN ZHU ${ }^{1 *}$, DEGUI WANG ${ }^{2 *}$, SHENQIAN LI $^{1 *}$, XUECHENG YANG $^{1}$, \\ YANWEI CAO $^{1}$, YONGHUA WANG ${ }^{1}$ and HAITAO NIU ${ }^{1}$ \\ ${ }^{1}$ Department of Urology, Affiliated Hospital of Qingdao University, Key Laboratory of Urinary System Diseases, \\ Qingdao, Shandong 266003; ${ }^{2}$ Department of Anatomy, School of Basic Medical Sciences, \\ Lanzhou University, Lanzhou, Gansu 730000, P.R. China
}

Received May 4, 2015; Accepted June 16, 2016

DOI: $10.3892 / 01.2016 .5047$

\begin{abstract}
The present study aimed to systematically analyze alterations in the expression of mitochondrial-associated proteins in human bladder cancer T24 cells co-cultured with tumor-associated human umbilical vein endothelial cells (HUVECs), and to investigate the characteristics of bladder cancer cell energy metabolism. The present study used the following techniques: A co-culture system of T24 cells and HUVECs was constructed using a microfluidic chip as a $3 \mathrm{D}$ co-culture system; the concentration of lactic acid in the medium of the cells was determined using an automatic microplate reader; a qualitative analysis of mitochondria-associated protein expression was performed by immunofluorescent staining; and a quantitative analysis of mitochondrial-associated protein expression was conducted using western blotting. The present results revealed that between the control groups (monoculture of T24 cells or HUVECs), the mitochondrial-associated protein fluorescence intensity was increased in the HUVECs compared with the T24 cells. The fluorescence intensity of mitochondrial-associated proteins in the HUVEC control group was increased compared with the HUVECs in the experimental co-culture group. In the T24 cells, the protein fluorescence intensity was increased in the experimental co-culture group compared with the control group. In addition, the expression of mitochondria-associated proteins was increased in HUVECs compared with T24 cells in the control groups, while T24 cells in the experimental co-culture group had an increased expression compared with HUVECs in the experimental group $(\mathrm{P}<0.05)$. For $\mathrm{T} 24$ cells, the expression of
\end{abstract}

Correspondence to: Dr Haitao Niu, Department of Urology, Affiliated Hospital of Qingdao University, Key Laboratory of Urinary System Diseases, 16 Jiangsu Road, Qingdao, Shandong 266003, P.R. China

E-mail: niuht0532@126.com

*Contributed equally

Key words: Warburg effect, 3D co-culture, metabolic coupling, tumor microenvironment, microfluidic chip mitochondrial-associated proteins was increased in the experimental group compared with the control group, and contrasting results were observed for the HUVECs $(\mathrm{P}<0.05)$. Determination of lactic acid concentration demonstrated that lactic acid concentration was highest in the experimental co-culture group, followed by the T24 control group and the HUVEC control group. In conclusion, the present study demonstrated that energy metabolism of the bladder tumor cells does not parallel the 'Warburg effect', since even under sufficient oxygen conditions the tumor cells still undergo glycolysis. Additionally, bladder tumor cells have an efficient oxidative phosphorylation process, wherein tumor cells promote glycolysis in adjacent interstitial cells, thereby causing increased formation of nutritional precursors. These high-energy metabolites are transferred to adjacent tumor cells in a specified direction and enter the Krebs Cycle. Ultimately, oxidative phosphorylation increases, and sufficient ATP is produced.

\section{Introduction}

Tumor cells do not exist as pure homogeneous populations in vivo. Instead, they are embedded in cancer cell nests, which are surrounded by stromal cells and extracellular matrix components, and these are important in the growth, invasion and metastasis of tumor cells. Consequently, endothelial cells and lactic acid affect almost every aspect of cancer progress, including the ability of tumor cells to grow and metastasize $(1,2)$. Therefore, it is a disadvantage to only investigate tumor cells in vitro. Glucose is the major energy-yielding nutrient and there are two primary ways for glycometabolism in cells: Glycolysis and oxidative phosphorylation (3). The majority of cells rely on oxidative phosphorylation to provide energy during well-oxygenated conditions; however, tumor cells depend on the glycolytic pathway for ATP generation even in an aerobic environment (4). Previously, in depth research concerning tumor metabolism has revealed that an increasing number of studies consider that the intermediate matrix produced by glycolysis during stromal cell metabolism may be used for synthesizing proteins, nucleic acids and lipids, which provide essential substrates for growth and metastasis of tumor cells $(1,2)$. The traditional Warburg Effect cannot explain all phenomenons that have been identified in 
recent studies; however the 'Reverse Warburg Effect' may be capable of doing this (5). Currently, studies concerning the Reverse Warburg Effect primarily focus on fibroblasts and macrophages; however, there is little study concerning tumor-associated endothelial cells in the tumor environment, which regulate growth, invasion and metastasis of tumor cells by producing various extracellular matrix and cytokines (6).

The effect of tumor-associated endothelial cells on the ability of tumor cells to grow and metastasize is not completely clear. Numerous studies hypothesize that in vivo tumor-associated endothelial cells receive biological signals from tumor cells and other tumor-associated stromal cells by absorbing paracrine factors, and subsequently induce tumor angiogenesis, which is essential for tumor growth and metastasis (7). In accordance with this hypothesis, the present study established a bionic 3D co-culture system using microfluidic chip technology in order to simulate the tumor microenvironment in vivo. The present study investigated the metabolic characteristics of tumor cells and tumor-endothelial metabolic coupling in the tumor microenvironment by measuring protein expression, which was determined by qualitative and quantitative analysis.

\section{Materials and methods}

\section{Materials}

Cells. Human bladder cancer T24 cells and human umbilical vein endothelial cells (HUVECs) were obtained from Central Laboratory, Affiliated Hospital of Qingdao University (Qingdao, China).

Antibodies and reagents. Mouse monoclonal anti-mitochondria antibody was purchased from Merck Millipore (Darmstadt, Germany; catalog no., MAB1273). The following antibodies were purchased from Abcam (Cambridge, UK): Rabbit polyclonal anti-translocase (outer mitochondrial membrane 20; catalog no., ab78547); rabbit polyclonal anti-mitofilin (mitochondiral inner membrane protein; catalog no., ab93323); rabbit polyclonal anti-cyclophilin (intercristal space protein; catalog no., ab3562); rabbit monoclonal anti-cytochrome $c$ (intermembrane space protein; catalog no., ab133504); rabbit polyclonal anti-NADH dehydrogenase subunit 6 (anti-oxidative phosphyorylation I; catalog no., ab81212); mouse monoclonal anti-mitochondrially encoded cytochrome $c$ oxidase I (anti-oxidative phosphyorylationIV; catalog no.,ab14705); rabbit polyclonal anti-ATP synthase (anti-oxidative phosphyorylation V; catalog no., ab96655); and mouse monoclonal anti-pyruvate dehydrogenase E1- $\alpha$ subunit (catalog no., ab110334). Rabbit monoclonal anti- $\beta$-actin (catalog no., 8457) and mouse monclonal anti- $\beta$-actin (catalog no., 3700) were purchased from Cell Signaling Technology, Inc. (Danvers, MA, USA). A goat polyclonal anti-rabbit secondary antibody conjugated to fluorescein isothiocyanate (FITC) (catalog no., BA1101) was purchased from Boster Biological Technology, Ltd. (Wuhan, China).

Dulbecco's modified Eagle's medium (DMEM), RPMI-1640 media, fetal bovine serum (FBS) and phosphate-buffered saline (PBS) were purchased from HyClone ${ }^{\mathrm{TM}}$ (GE Healthcare Life Sciences, Logan City, UT, USA). MitoTracker ${ }^{\circledR}$ Red CMXRos was purchased from Gibco ${ }^{\circledR}$ (Thermo Fisher Scientific, Inc., Waltham, MA, USA; catalog no., M7512). 4',6-diamidino-2-phenylindole (DAPI; catalog no., AR1177) and BCA Protein Assay kit (catalog no., AR0146) were purchased from Boster Biological Technology, Ltd. An L-Lactic Acid Assay kit was purchased from Seebio Biotech, Inc. (Shanghai, China; catalog no., K-DLATE). A Simon ${ }^{\mathrm{TM}}$ (12-180 kDa) Master kit was purchased from ProteinSimple (San Jose, CA, USA), which included a biotinylated molecular weight ladder, streptavidin-horseradish peroxidase (HRP), fluorescent standards, luminol-S, hydrogen peroxide, sample buffer, DTT, stacking matrix, separation matrix, running, buffer and matrix removal buffers, antibody diluent, goat anti-rabbit and anti-mouse secondary antibodies, and capillary clips.

Analytical instruments. An automatic microplate reader (Bio-Tek Instruments, Inc., Winooski, VT, USA), a fluorescence microscope (Nikon Corporation, Tokyo, Japan) and Simple Westerns System (ProteinSimple) were used in the present study.

\section{Methods \\ Microfluidic chip technology}

Designing and producing a microfluidic chip with a multi-channel connection and a multi-unit integrated high-throughput system. The chip consisted of the following components: Cell culture pools, microchannels connected to the pools and peripheral perfusion channels. In detail, each chip had four cell culture pools: Two pools were inoculated with HUVECs and the other two pools were inoculated with T24 cells. Each culture pool had two channels connected to the outside. One pool was the in-channel for cell seeding, and one was the out-channel. The four culture pools were connected by two cross microchannels impregnated with Matrigel, which separated the cells in each pool; however, lactic acid and other small molecules produced by the cells could be exchanged via the Matrigel. A perfusion channel was located around the entire periphery of the chip. Cell culture medium was injected through the perfusion channel, whereas cells were seeded into the pools (8) (Fig. 1).

Experimental groups (Fig. 1). Experimental co-culture group: Co-culture of T24 cells and HUVECs in a microfluidic chip. T24 cells were perfused into chamber A and D, and HUVECs were perfused into chamber B and C. Control group 1: Monoculture of T24 cells in a microfluidic chip. T24 cells were perfused into chamber A-D. Control group 2: Monoculture of HUVECs in a microfluidic chip. HUVECs were perfused into chamber A-D.

Matrigel preparation and perfusion. To ensure that the prepolymer matrix solution was able to dissolve, the solution was maintained in a $4^{\circ} \mathrm{C}$ refrigerator overnight. The microfluidic chip and pipette were pre-cooled during this time. Subsequently, serum-free culture medium and pre-cooled melted Matrigel were mixed at a ratio of $1: 1(\mathrm{v} / \mathrm{v})$ and $10 \mu \mathrm{l}$ was pipetted into the microfluidic chips via the cross microchannel. The microfluidic chip was then placed in a sterile petri dish in a $37^{\circ} \mathrm{C}$ incubator for $\geq 8 \mathrm{~h}(9)$.

Cell line culturing. T24 cells were cultured in RPMI-1640 medium with $10 \%$ FBS, and HUVECs were cultured in DMEM with $10 \%$ FBS. T24 cells and HUVECs that grew well and possessed ideal cell morphology were selected for experiments, in order to prepare a high-density cell suspension 
A

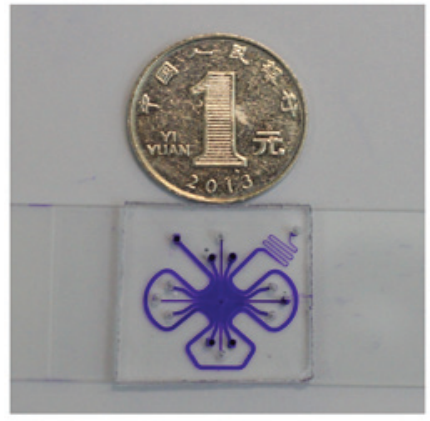

C

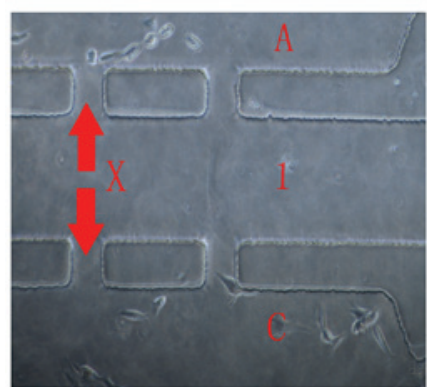

B
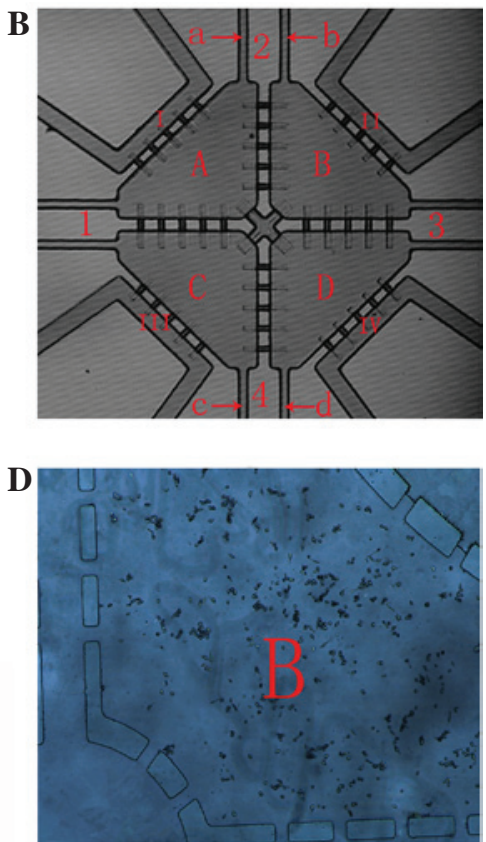

Figure 1. Bionic 3D co-culture microsystem. (A) A microfluidic chip is as large as a coin. (B) A total of $10 \mu 1$ mixed solution is injected into the microfluidic chip via channels 1-4. A previously prepared cell suspension and Matrigel solution are inserted into the chip via channels a-d. Complete medium is inserted into the microfluidic chip via channels I-IV (perfusion channels). Cells are grown in chambers A-D. (C) Human bladder cancer T24 cells and human umbilical vein endothelial cells communicate with each other via the channel marked X. (D) Representative cells grown in chamber B.

$\left(10^{5}\right.$ cells $\left./ \mathrm{ml}\right)$. The cell suspension was precooled on ice prior to mixing with RPMI-1640 and DMEM media with $10 \%$ FBS at a ratio of $3: 1(\mathrm{v} / \mathrm{v})$. Each cell culture pool was injected with $5 \mu \mathrm{l}$ of the cell suspension. T24 cells and HUVECs were seeded in the pools using a diagonal-cross method in the experimental groups, whereas a single cell type was seeded in all the pools in the control groups. RPMI-1640 or DMEM media were injected into the perfusion channel, completing the microfluidic chip laboratories. The chips were placed in a $37^{\circ} \mathrm{C}$ incubator with $5 \% \mathrm{CO}_{2}$ for $48 \mathrm{~h}$.

Determination of the lactic acid content of the culture broth. After culturing the microfluidic chips for $48 \mathrm{~h}$ at $37^{\circ} \mathrm{C}$, the medium was removed from each pool using a micropipette and centrifuged for $5 \mathrm{~min}$ at 1,119 x g. Subsequently, a L-Lactic Acid Assay kit was used, according to the manufacturer's protocol. Briefly, $3 \mu$ l lactic acid concentration calibrator was added with $240 \mu \mathrm{l}$ reagent 1 (Tris-buffer and lactic acid oxidase). The resulting solution was incubated for $5 \mathrm{~min}$ at $37^{\circ} \mathrm{C}$, and the first photometric value (A1) was analyzed. Subsequently, $60 \mu$ l reagent 2 (peroxidase, 4-aminoantipyrine and TBHBA) was added. After a $5 \mathrm{~min}$ incubation at $37^{\circ} \mathrm{C}$, the second photometric value (A2) was analyzed. The change in photometric value for each tube was calculated as follows: $\Delta \mathrm{A}=\mathrm{A}_{2}-\mathrm{A}_{1}$. The following equation was used to calculate the lactic acid content: $\mathrm{C}=\Delta \mathrm{AU} \times \mathrm{CS} / \triangle \mathrm{AS}$, where $\mathrm{CS}$ represents the concentration of the calibration solution, $U$ is the medium (ml) and $\mathrm{S}$ is the lactic acid concentration calibrator.

Qualitative analysis of mitochondria-associated proteins using immunofluorescence. A total of $5 \mu \mathrm{l}$ MitoTracker Red ( $200 \mathrm{nmol} / \mathrm{l})$ was added to each cell pool, and the pools were incubated for $45 \mathrm{~min}$ in a $37^{\circ} \mathrm{C}$ incubator. Subsequently,

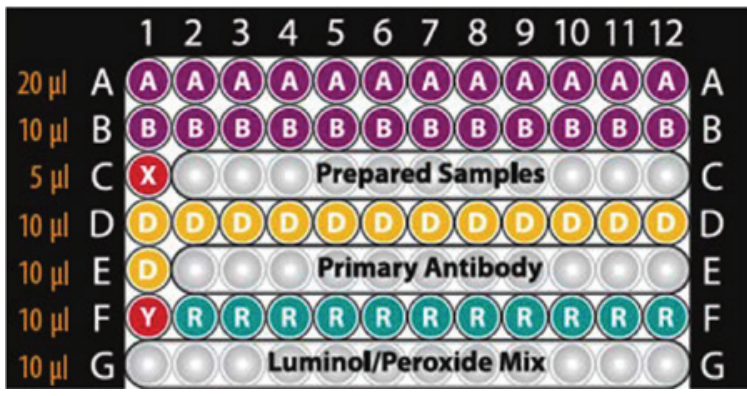

Figure 2. Schematic of the Simple Western ${ }^{\text {TM }}$ plate (ProteinSimple) used in the present study. Sample, rows A-G; streptavidin-HRP, well Y; goat anti-rabbit HRP, well R; antibody diluent Plus, well D; biotinylated ladder, well X; Stacking Matrix 1, well B; Separation Matrix 1, well A. HRP, horseradish peroxidase.

fixation and permeabilization was performed according to the immunofluorescence protocol (10). The primary antibody used was a mouse monoclonal anti-mitochondria antibody, and the secondary antibody was conjugated to FITC. After washing the pools with PBS, $5 \mu$ l DAPI was added to each pool for $30 \mathrm{~min}$ and then each pool was washed. All of the above steps were performed in the dark. Fluorescence intensity was observed using immunofluorescence microscopy. MitoTracker Red is excited by green light, anti-mitochondria antibody by blue light, and DAPI by ultraviolet light.

Quantitative analysis of mitochondria-associated proteins in using western blotting. Mitochondrial proteins were assessed using Simple Westerns ${ }^{\text {TM }}$ System from ProteinSimple, which is more advanced and accurate compared with normal western blotting. Compared with western blotting, Simple Westerns has a shorter operation time, fewer sample proteins and a higher 

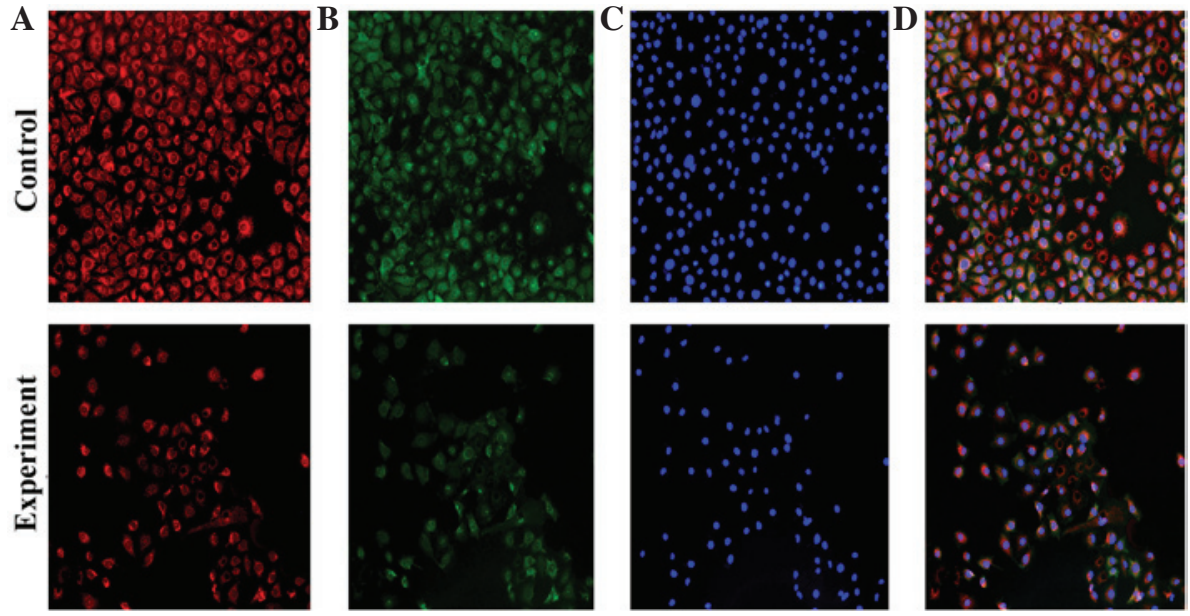

Figure 3. Immunofluorescence of human umbilical vein endothelial cells in a microfluidic chip from a control (monoculture) group and an experimental co-culture group with human bladder cancer T24 cells. (A) MitoTracker Red staining. (B) Anti-mitochondria antibody green staining. (C) 4,6-diamidino-2-phenylindole staining. (D) Image merging.

protein flux (run 12 proteins at a time), so that accurate results are obtained, which have less errors and a better comparison. The reagents used were as follows: Antibody diluent plus, sample buffer 10X, separation Matrix 1, stacking matrix 1, streptavidin-HRP, goat-anti-rabbit HRP-conjugated secondary antibody, matrix removal buffer, wash buffer, running buffer and DTT (DTT is used to reduce samples during denaturation). Samples were prepared by extracting mitochondrial proteins using a modified RIPA buffer [50 mM Tris (pH 7.4), $150 \mathrm{mM}$ $\mathrm{NaCl}, 0.1 \%$ SDS, $0.25 \%$ sodium deoxycholate, $2 \%$ TritonX100, $1 \mathrm{mM}$ PMSF, $2 \mu \mathrm{M}$ leupeptin], subsequent to the $48 \mathrm{~h}$ incubation of the experimental co-culture and control microfluidic chips. Protein concentrations were $>2 \mu \mathrm{g} / \mu \mathrm{l}$, as assessed using the BCA Protein Assay kit. The protein extraction and the sample diluent were mixed to reduce protein concentrations to $1.5 \mu \mathrm{g} / \mu \mathrm{l}$. Subsequently, a total of $1.25 \mu \mathrm{l}$ Fluorescent Standard/4X Master Mix was combined with $3.75 \mu$ l protein extract in a fresh microfuge tube for each preparation. This was vortexed and denatured by heating to $95^{\circ} \mathrm{C}$ for $5 \mathrm{~min}$ using a heat block. The sample was vortexed again, microcentrifuged $(1,119 \times \mathrm{g})$ and stored on ice until ready to use.

Sample plates were prepared as follows: The reagent and sample were placed on a 384-well microplate; the reagents were added to the assay plate using the volume guidelines listed in Fig. 2; the assay plate was covered with a lid and centrifuged at $6,994 \mathrm{x} \mathrm{g}$ for $5 \mathrm{~min}$ at room temperature to remove any air bubbles in the plate wells. Following this the plate was run in the Simon ${ }^{\mathrm{TM}}$ System. Removal, wash and running buffers were added to the system tray, and a capillary clip was inserted into the clip holder. Subsequently, the lid covering the assay plate was removed and Compass version 1.7 software (ProteinSimple) was run. Antibodies were diluted to 1:200. The samples were divided into two groups: Group 1 consisted of the mitochondrial outer membrane protein (channel protein subtype), mitochondrial inner membrane protein (Core 1), intracristal space protein (cyclophilin D) and intermembrane space protein (cytochrome C); group 2 consisted of anti-oxidative phosphorylation I (NADH dehydrogenase), anti-oxidative phosphorylation IV (cytochrome $c$ oxidase), anti-oxidative phosphorylation V (ATP synthase) and pyruvate dehydrogenase. Data analysis was performed using Compass software.

Statistical analysis. Statistical analysis was performed using SPSS version 17.0 software (SPSS, Inc., Chicago, IL, USA). Student's t-test was used to evaluate potential associations in tumor cells, HUVECs and cells in the co-culture group. $\mathrm{P}<0.05$ was considered to indicate a statistically significant difference.

\section{Results}

Qualitative analysis. MitoTracker Red and anti-mitochondria antibody were used to observe mitochondria. The former stains living cells and the latter stain cells following fixation and permeabilization. With different fluorescence excitation, fluorescence images are obtained that have the same exposure time, contrast and enlargement factor. Fluorescence intensity reflects the amount of mitochondrial proteins, and the amount of fluorescent cells reflect cell proliferation and survival capability. Fig. 3 shows that the fluorescence intensity of HUVECs in control group is increased compared with the HUVECs in the experimental co-culture group. Similarly, the amount of fluorescent HUVECs in the control group is increased compared with HUVECs in the experimental group. This suggests that when T24 cells are cultured with HUVECs, the mitochondrial aerobic capacity, and cell proliferation and survival capability of HUVECs are decreased. Fig. 4 shows the fluorescence intensity of T24 cells in the experimental co-culture group is increased compared with the T24 cells in the control group. In addition, the amount of fluorescent T24 cells in the experimental co-culture group is increased compared with T24 cells in the control group. This suggests that when T24 cells are cultured with HUVECs, the mitochondrial aerobic capacity, and cell proliferation and survival capability of the T24 cells are increased.

Quantitative analysis. Western blot images (Fig. 5) were obtained subsequent to running a Simple Western. In order to enhance the accuracy of the results, every western blot was repeated 3 times. Outcomes are shown in Fig. 5. In 

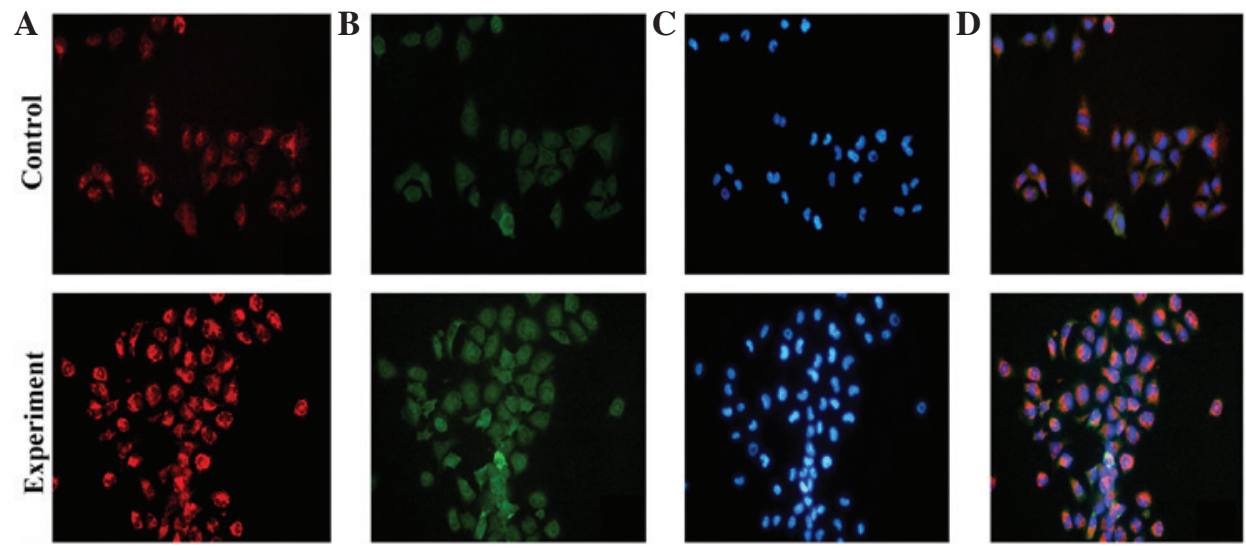

Figure 4. Immunofluorescence of human bladder cancer T24 cells in a microfluidic chip from a control (monoculture) group and an experimental co-culture group with human umbilical vein endothelial cells. (A) MitoTracker Red staining. (B) Anti-mitochondria antibody green staining. (C) 4,6-diamidino-2-phenylindole staining. (D) Image merging.

the control groups, total protein expression of T24 cells is clearly decreased compared with HUVECs $(\mathrm{P}=0.038)$. In the experimental co-culture group, total protein expression in T24 cells is increased compared with HUVECs $(\mathrm{P}=0.020)$. Total protein expression in T24 cells was clearly increased in the experimental group compared with the T24 cell control group $(\mathrm{P}=0.002)$. By contrast, total protein expression in the HUVECs in the experimental group was decreased compared with the HUVEC control group ( $\mathrm{P}=0.029)$.

Lactic acid concentrations. Fig. 6 shows that in the control group lactic acid concentration of the T24 cell lysis solution was $4.17 \mathrm{~mol} / \mathrm{ml}$, and the lactic acid concentration of the HUVEC lysis solution was $4.41 \mathrm{~mol} / \mathrm{ml}$. In the experimental group, lactic acid concentration of the total cell lysis solution was $4.92 \mathrm{~mol} / \mathrm{ml}$. These results are the mean value of three independent experiments. This result suggests that cells in the co-culture experimental group have a higher level of aerobic glycolysis compared with the control group.

\section{Discussion}

Under conditions of adequate oxygen supply, normal cells undergo efficient oxidative phosphorylation. Glucose-derived pyruvate is transported into the mitochondria, where it undergoes oxidative decarboxylation to produce acetyl coenzyme A via pyruvate dehydrogenase. Acetyl coenzyme A enters the tricarboxylic acid cycle, which consists of numerous steps, including oxidation, that ultimately generate water, carbon dioxide and significant amounts of ATP. In conditions of hypoxia or a reduced oxygen supply, cellular energy metabolism is chiefly dependent on the glycolytic pathway, which is predominantly performed in the cytoplasm (11). To a considerable extent, research into mitochondria metabolism is similar to research into cellular energy metabolism, in that alterations in protein expression are equal to alterations in cellular energy metabolism. According to the present experimental results, there is accurate metabolic coupling between tumor and tumor-associated endothelial cells in the tumor microenvironment. Protein expression in the experimental T24 cells was significantly increased compared with T24 cells in the control group. In addition, protein expression in HUVECs

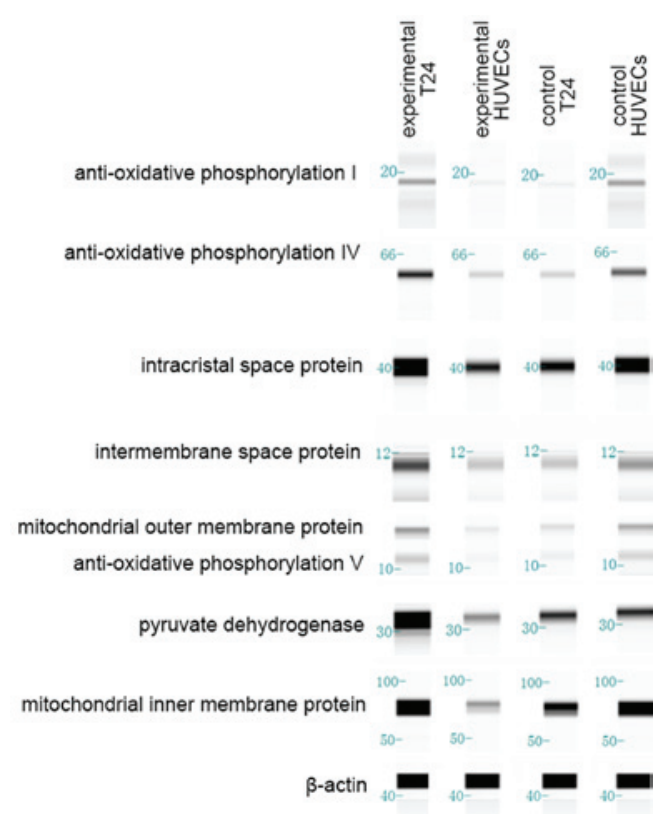

Figure 5. Western blot images obtained subsequent to running a Simple Western $^{\mathrm{TM}}$ System (ProteinSimple). In the control monoculture groups, protein expression of human bladder cancer T24 cells was decreased compared with HUVECs. In the experimental co-culture groups, protein expression of T24 cells was increased compared with HUVECs. In T24 cells, protein expression was increased in the experimental group compared with the control group. In HUVECs, protein expression was decreased in the experimental group compared with the control group. HUVECs, human umbilical vein endothelial cells.

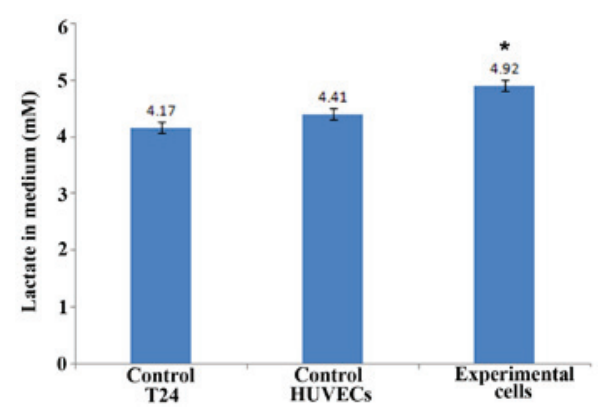

Figure 6. Lactic acid concentration of human bladder cancer T24 cells and HUVECs lysis solution in the control monoculture group, and the experimental co-culture group. " $\mathrm{P}<0.05$ vs control T24 cells and control HUVECs. HUVECs, human umbilical vein endothelial cells. 
in the experimental group was decreased compared with HUVECs in the control group (Fig. 5). These results suggest that tumor-associated endothelial cells promote the oxidative phosphorylation of tumor cells. By contrast, tumor cells restrain the oxidative phosphorylation of endothelial cells. Additionally, fluorescence intensity and the amount of fluorescent cells in the experimental co-culture group was increased compared with the control group in tumor cells; however, this was reversed in HUVECs (Figs. 3 and 4). This supports the conclusion that tumor-associated endothelial cells promote the oxidative phosphorylation of tumor cells, while tumor cells restrain the oxidative phosphorylation of endothelial cells. The present conclusion differs from traditional tumor energy metabolism, which considers that tumor cells primarily rely on the aerobic glycolytic pathway for ATP generation.

MitoTracker Red is a living cell fluorochrome, and results observed with this staining technique may directly reflect cell reproductive activity. In the present study, the cells grew well in the microfluidic chips (Figs. 3 and 4). Tumor cells in the experimental group exhibited the highest cell viability, with HUVECs in the experimental group exhibiting the second highest viability. This result demonstrates that in the co-culture system, these two types of cell interact and aid each other. In the present quantitative analysis, mitochondrial outer membrane protein, mitochondrial inner membrane protein, intracristal space protein and intermembrane space protein were all expressed by the cells. This revealed that co-culture in the microfluidic chip did not affect the integrity of mitochondrial structure. Anti-oxidative phosphorylation I, IV and V proteins were also expressed by the cells, which demonstrated that co-culture in the microfluidic chip did not affect the integrity of mitochondrial function. Tumor cells in the experimental group exhibited the highest protein expression, and HUVECs in the experimental group exhibited the lowest protein expression (Fig. 5). These results suggest that in the co-culture system the majority of the energy for tumor cells is obtained from oxidative phosphorylation; however, for HUVECs the majority of energy is obtained from aerobic glycolysis. This creates a strong positive feedback loop: Oxidative phosphorylation of tumor cells and aerobic glycolysis of HUVECs aid each other. This positive feedback may be a reason by which tumor growth and metastasis is continuously promoted.

The primary mechanism of tumor cell growth in the tumor microenvironment may be as follows: i) During tumor growth and metastasis, tumor-associated endothelial cells produce certain cell factors, and these factors promote oxidative phosphorylation of tumor cells, thus providing essential energy for cell growth. In addition, these factors restrain glycometabolism of stromal cells. ii) Primarily, HUVECs obtain energy from aerobic glycolysis, even under well-oxygenated conditions (12); therefore, lactic acid produced by aerobic glycolysis enters tumor cells as metabolic matter by the energy transfer pathway, which increases the substrate concentration of oxidative phosphorylation, leading to the promotion of oxidative phosphorylation of tumor cells. Consequently, the present study hypothesizes that tumor-associated endothelial cells grow out of tumor tissues first. However, this is without a blood supply, and endothelial cells gain energy from glycolysis. The production of glycolysis enters into tumor cells for aerobic glycolysis, thus providing steady energy for growth of tumor cells. As endothelial cells grow out of tumor issues, vascularization supplies oxygen, which is required for oxidative phosphorylation. Glycolysis is a fast way to produce energy, which leads to a rapid growth of endothelial cells. Consequently, endothelial cell growth leads to tumor cell growth, and tumor cells promote the glycolysis of endothelial cells. This interactive mechanism may explain why the growth rate of certain tumor tissues is faster than other types. iii) Certain studies have revealed that tumor cells induce oxidative stress in stromal cells, thus activating the nuclear factor- $\kappa \mathrm{B}$ pathway, hypoxia-inducible factor-1 and reactive oxygen species, which induce each other and cause endothelial cell autophagy and mitochondrial autophagy (13-16). This produces numerous substrates for oxidative phosphorylation, therefore providing a large amount of continuous energy for tumor cell growth and metastasis. There is a certain amount of positive feedback regulation between tumor cells and tumor-associated endothelial cells. Oxidative stress caused by tumor cells is the trigger of this positive feedback. Once the trigger is induced, tumor cells continuously obtain substrates for oxidative phosphorylation from stromal cells, thus gaining energy from oxidative phosphorylation. To a certain extent, this mechanism may explain unlimited tumor growth, and this mechanism, which is known as oxidative stress energy theory (17), may also explain why when tumors metastasize, tumor cells maintain rapid growth, even without a substantial blood supply.

Clinically, autophagy inducers and inhibitors are effective anti-cancer therapies, which is known as the autophagy paradox. Inducers of autophagy, including temsirolimus and everolimus, have been demonstrated to be effective therapies for renal cell carcinoma $(18,19)$ and glioma $(20)$. Chloroquine, an autophagy inhibitor has prolonged the survival of patients with glioblastoma multiforme (21), and is currently being studied in clinical trials for other malignancies. The novel theory presented in the current study may explain this paradox; the systemic induction of autophagy prevents tumor cells from using recycled nutrients, while the systemic inhibition of autophagy prevents stromal cells from producing recycled nutrients, leading to tumor cell starvation. Therefore, energy transfer from the tumor stroma to tumor cells may be used to explain the autophagy paradox. The present study revealed that lactic acid concentrations in the tumor cells experimental co-culture system were significantly increased compared with the control group. Clearly, the reason for a high lactic acid concentration is that tumor cells promote aerobic glycolysis of endothelial cells and trigger endothelial cell autophagy. Therefore, lactic acid enters tumor cells as a substrate of oxidative phosphorylation and allows the growth of tumor cells.

In conclusion, the present study investigated tumor-endothelial metabolic coupling in the tumor microenvironment and revealed that lactic acid is the primary link. Even though a high lactic acid concentration was demonstrated to be the factor for oxidative phosphorylation in tumor cells, the present study did not verify whether lactic acid is the only factor. Therefore, in future studies small interfering RNA may be used to block certain glycolysis pathways to identify the factor for oxidative phosphorylation of tumor cells. Clinically, through competitive inhibition and blocking of this pathway, the source of energy to tumor cells may be reduced and inhibited, thus inhibiting the growth of tumors from a metabolic perspective. 


\section{Acknowledgments}

The present study was supported by the National Natural Science Foundation of China (Beijing, China; grant nos. 30901481, 81372752 and 81472411), Wu JiePing Medical Foundation (Beijing, China; grant no. 320.6750.13261) and Natural Science Foundation of Shandong Province (Jinan, China; grant no. ZR2014HM088).

\section{References}

1. Gonda TA, Tu S and Wang TC: Chronic inflammation, the tumor microenvironment and carcinogenesis. Cell Cycle 8: 2005-2013, 2009.

2. Boelens MC, Wu TJ, Nabet BY, Xu B, Qiu Y, Yoon T, Azzam DJ, Twyman-Saint Victor C, Wiemann BZ, Ishwaran $\mathrm{H}$, et al: Exosome transfer from stromal to breast cancer cells regulates therapy resistance pathways. Cell 159: 499-513, 2014.

3. Zala D, Hinckelmann MV, Yu H, Lyra da Cunha MM, Liot G, Cordelières FP, Marco S and Saudou F: Vesicular glycolysis provides on-board energy for fast axonal transport. Cell 152 479-491, 2013.

4. Vander Heiden MG, Cantley LC and Thompson CB: Understanding the Warburg effect: The metabolic requirements of cell proliferation. Science 324: 1029-1033, 2009.

5. Martinez-Outschoorn UE, Whitaker-Menezes D, Valsecchi M, Martinez-Cantarin MP, Dulau-Florea A, Gong J, Howell A, Flomenberg N, Pestell RG, Wagner J, et al: Reverse Warburg effect in a patient with aggressive B-cell lymphoma: Is lactic acidosis a paraneoplastic syndrome? Semin Oncol 40: 403-418, 2013.

6. Carmona-Fontaine C, Bucci V, Akkari L, Deforet M, Joyce JA and Xavier JB: Emergence of spatial structure in the tumor microenvironment due to the Warburg effect. Proc Natl Acad Sci USA 110: 19402-19407, 2013.

7. Jean C, Chen XL, Nam JO, Tancioni I, Uryu S, Lawson C, Ward KK, Walsh CT, Miller NL, Ghassemian M, et al: Inhibition of endothelial FAK activity prevents tumor metastasis by enhancing barrier function. J Cell Biol 204: 247-263, 2014.

8. Tang J, Cui J, Chen R, Guo K, Kang X, Li Y, Gao D, Sun L, $\mathrm{Xu} \mathrm{C}$, Chen J, et al: A three-dimensional cell biology model of human hepatocellular carcinoma in vitro. Tumor Biol 32: 469-479, 2011

9. Shiraki N, Yamazoe T, Qin Z, Ohgomori K, Mochitate K, Kume K and Kume S: Efficient differentiation of embryonic stem cells into hepatic cells in vitro using a feeder-free basement membrane substratum. PLoS One 6: e24228, 2011.
10. Huh D, Matthews BD, Mammoto A, Montoya-Zavala M, Hsin HY and Ingber DE: Reconstituting organ-level lung functions on a chip. Science 328: 1662-1668, 2010.

11. Schulz TJ, Thierbach R, Voigt A, Drewes G, Mietzner B, Steinberg P, Pfeiffer AF and Ristow M: Induction of oxidative metabolism by mitochondrial frataxin inhibits cancer growth: Otto Warburg revisited. J Biol Chem 281: 977-981, 2006.

12. De Bock K, Georgiadou M, Schoors S, Kuchnio A, Wong BW, Cantelmo AR, Quaegebeur A, Ghesquière B, Cauwenberghs S, Eelen G, et al: Role of PFKFB3-driven glycolysis in vessel sprouting. Cell 154: 651-663, 2013.

13. Martinez-Outschoorn UE, Pavlides S, Howell A, Pestell RG, Tanowitz HB, Sotgia F and Lisanti MP: Stromal-epithelial metabolic coupling in cancer: Integrating autophagy and metabolism in the tumor microenvironment. Int J Biochem Cell Biol 43: 1045-1051, 2011.

14. Ghosh S, Mukherjee S, Choudhury S, Gupta P, Adhikary A, Baral R and Chattopadhyay S: Reactive oxygen species in the tumor niche triggers altered activation of macrophages and immunosuppression: Role of fluoxetine. Cell Signal 27: 1398-1412, 2015.

15. Lee M and Yoon JH: Metabolic interplay between glycolysis and mitochondrial oxidation: The reverse Warburg effect and its therapeutic implication. World J Biol Chem 6: 148-161, 2015.

16. Meseure D, Drak Alsibai K and Nicolas A: Pivotal role of pervasive neoplastic and stromal cells reprogramming in circulating tumor cells dissemination and metastatic colonization. Cancer Microenviron 7: 95-115, 2014.

17. Martinez-Outschoorn UE, Balliet RM, Rivadeneira DB, Chiavarina B, Pavlides S, Wang C, Whitaker-Menezes D, Daumer KM, Lin Z, Witkiewicz AK, et al: Oxidative stress in cancer associated fibroblasts drives tumor-stroma co-evolution: A new paradigm for understanding tumor metabolism, the field effect and genomic instability in cancer cells. Cell Cycle 9: 3256-3276, 2010

18. Hudes G, Carducci M, Tomczak P, Dutcher J, Figlin R, Kapoor A, Staroslawska E, Sosman J, McDermott D, Bodrogi I, et al: Temsirolimus, interferon alfa, or both for advanced renal-cell carcinoma. N Engl J Med 356: 2271-2281, 2007.

19. Motzer RJ, Escudier B, Oudard S, Hutson TE, Porta C, Bracarda S, Grünwald V, Thompson JA, Figlin RA, Hollaender N, et al: Efficacy of everolimus in advanced renal cell carcinoma: A double-blind, randomised, placebo-controlled phase III trial. Lancet 372: 449-456, 2008.

20. Krueger DA, Care MM, Holland K, Agricola K, Tudor C, Mangeshkar P, Wilson KA, Byars A, Sahmoud T and Franz DN: Everolimus for subependymal giant-cell astrocytomas in tuberous sclerosis. N Engl J Med 363: 1801-1811, 2010.

21. Sotelo J, Briceño E and López-González MA: Adding chloroquine to conventional treatment for glioblastoma multiforme: A randomized, double-blind, placebo-controlled trial. Ann Intern Med 144: 337-343, 2006. 